NISSUNA UMANA INVESTIGAZIONE SI PUO DIMANDARE VERA SCIENZIA S'ESSA NON PASSA PER LE MATEMATICHE DIMOSTRAZIONI LEONARDO DA VINCI

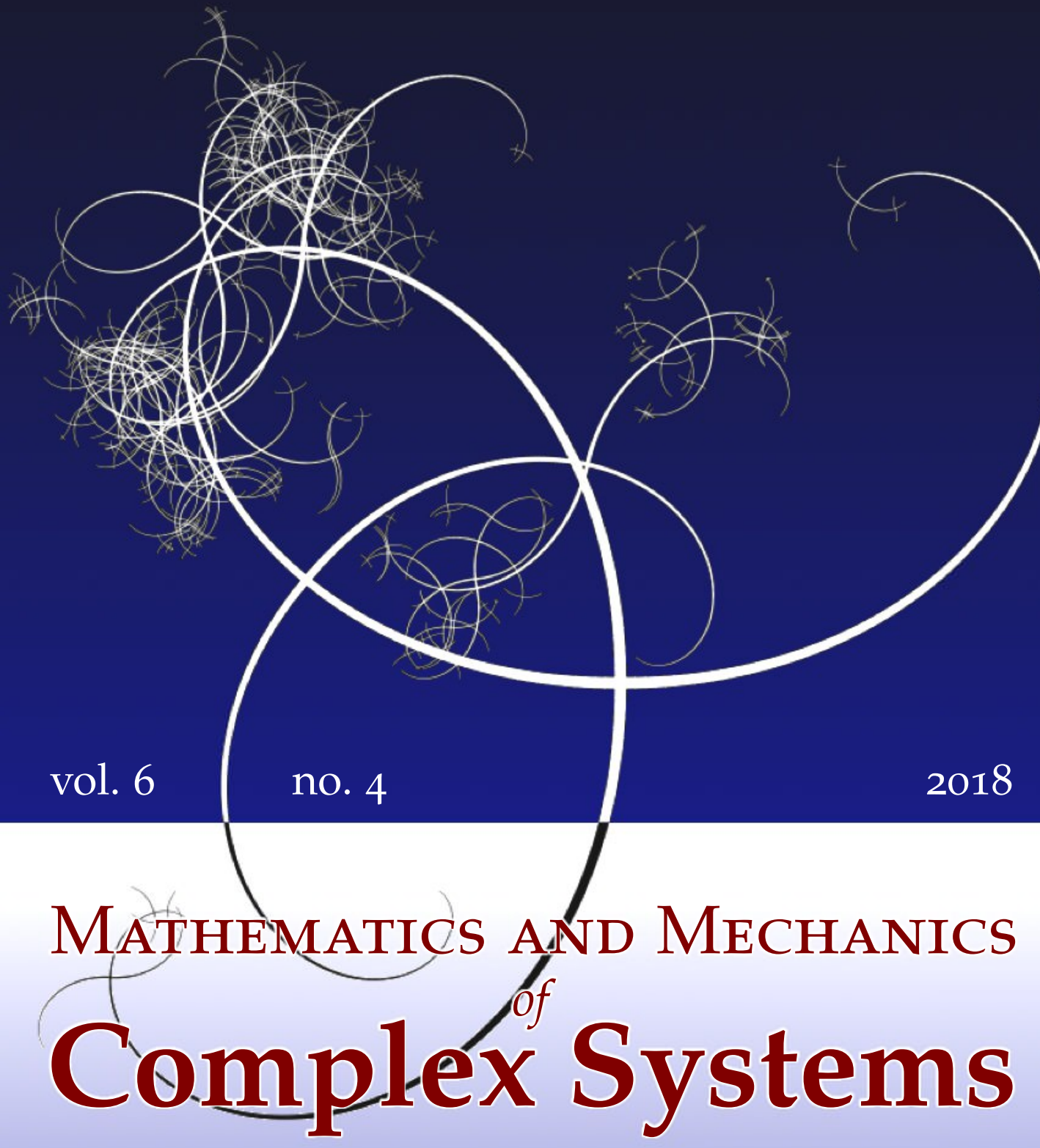

Christophe Cluzel and Rachele Allena

A GENERAL METHOD FOR THE DETERMINATION OF THE LOCAL ORTHOTROPIC DIRECTIONS OF HETEROGENEOUS MATERIALS:

APPLICATION TO BONE STRUCTURES USING $\mu$ CT IMAGES 


\title{
A GENERAL METHOD FOR THE DETERMINATION OF THE LOCAL ORTHOTROPIC DIRECTIONS OF HETEROGENEOUS MATERIALS: APPLICATION TO BONE STRUCTURES USING $\mu$ CT IMAGES
}

\author{
Christophe Cluzel and Rachele Allena
}

\begin{abstract}
To assess the degree (i.e., isotropy, transverse isotropy, or orthotropy) and the directions of anisotropy of a three-dimensional structure, information about its mesostructure is necessary. Usually, a topological analysis of computed tomography or microcomputed tomography images is performed and requires an interpretation of the constitutive elements of the three-dimensional structure, which may lead to a simplistic description of the geometry. In this paper we propose an alternative technique based on a geometric tensor and we use it to analyze 38 representative elementary volumes extracted from 24 specimens of cortical bone in a human femur whose geometries have been reconstructed via microcomputed tomography images.
\end{abstract}

\section{Introduction}

Computed tomography $(\mathrm{CT})$ and microcomputed tomography $(\mu \mathrm{CT})$ are powerful imaging tools allowing the visualization of three-dimensional (3D) geometries which can be used to simulate the global and personalized response of the mechanical structure [Rémond et al. 2016]. If such geometries are constituted of heterogeneous materials like bone or composites [Placidi et al. 2017; Giorgio et al. 2017], one needs to describe their constitutive behavior as a function of the local systems of anisotropy. Then, additional information is required at the scale of their mesostructure to identify the anisotropic field.

Cortical bone is constituted of several elements oriented in space leading to a very significant anisotropy at different levels, from the nanoscale (i.e., collagen fibers) to the mesoscale (i.e., osteons) [Rho et al. 1998]. As a consequence, the elastic behavior at the macroscale is highly anisotropic and more specifically orthotropic as has been quantified in [Rho 1996; Bernard et al. 2013].

The identification of the directions of orthotropy may be straightforward and given by the direct observation of the Haversian canals. For instance, in [Heřt

Keywords: orthotropic materials, cortical bone, $\mu \mathrm{CT}$ images, geometric tensor. 
et al. 1994; Petrtýl et al. 1996], the canals are previously ink-soaked and then developed by successive polishing. A similar technique has also been adopted by Báča et al. [2007] to describe the directions of the canals on the bone surface.

Alternatively, a topological analysis of CT or $\mu \mathrm{CT}$ images can be employed to identify the degree (i.e., isotropy, transverse isotropy, or orthotropy) and the main directions of anisotropy after a 3D skeletonization as in [Pothuaud et al. 2000] or a 3D finite element (FE) simulation as in [Nazemi et al. 2016], both applied on trabecular bone. Nonetheless, such an approach requires a complex interpretation of the constitutive elements of the 3D structure. Therefore, in this paper we propose an alternative technique based on a geometric tensor and we use it to analyze a series of representative elementary volumes (REVs) extracted from cortical bone specimens and whose 3D geometries are obtained via $\mu \mathrm{CT}$ images. Assuming an orthotropic elastic behavior for the cortical bone, the average directions of the mesostructure are computed.

In the following sections we describe the experimental approach used to identify the main directions of orthotropy of the cortical bone mesostructure. This includes the specimen extraction (Section 2.1.1), the $\mu \mathrm{CT}$ imaging (Section 2.1.2), and the computation of the geometrical tensor associated with the femur mesostructure (Section 2.1.3). In Section 3, we first show the consistency of the technique to identify the directions of orthotropy through simple geometric configurations (Section 3.1) and second we apply our approach on the bone specimens (Section 3.2). Finally, in Section 4, the results are discussed and some limitations and perspectives of the work are considered.

\section{Material and methods}

\subsection{Experimental analysis of the orthotropic field.}

2.1.1. CT-scanning and specimen extraction. A left human male femur (91 years old) was collected and frozen at $-20^{\circ} \mathrm{C}$ in a plastic bag. Once defrosted, the femur was cleaned by a clinician to remove soft tissues around it and dried with ethanol.

A total of 24 specimens $S_{i}$, with $i$ from 1 to 24 , were extracted at different regions of the proximal side of the femur as follows (Figure 1):

- 3 along the upper anterior diaphysis (AD) ( $S_{1}$ to $\left.S_{3}\right)$,

- 2 in the greater trochanter (GT) $\left(S_{4}\right.$ and $\left.S_{5}\right)$,

- 4 around and on top of the lesser trochanter (LT) ( $S_{6}$ to $S_{9}$ ),

- 3 along the femoral neck (N) ( $S_{10}$ to $\left.S_{12}\right)$,

- 2 in the femoral head (H) $\left(S_{13}\right.$ and $\left.S_{14}\right)$,

- 4 in the upper posterior diaphysis (PD) $\left(S_{15}\right.$ to $\left.S_{18}\right)$, and

- 6 around the diaphysis (D) ( $S_{19}$ to $\left.S_{24}\right)$. 


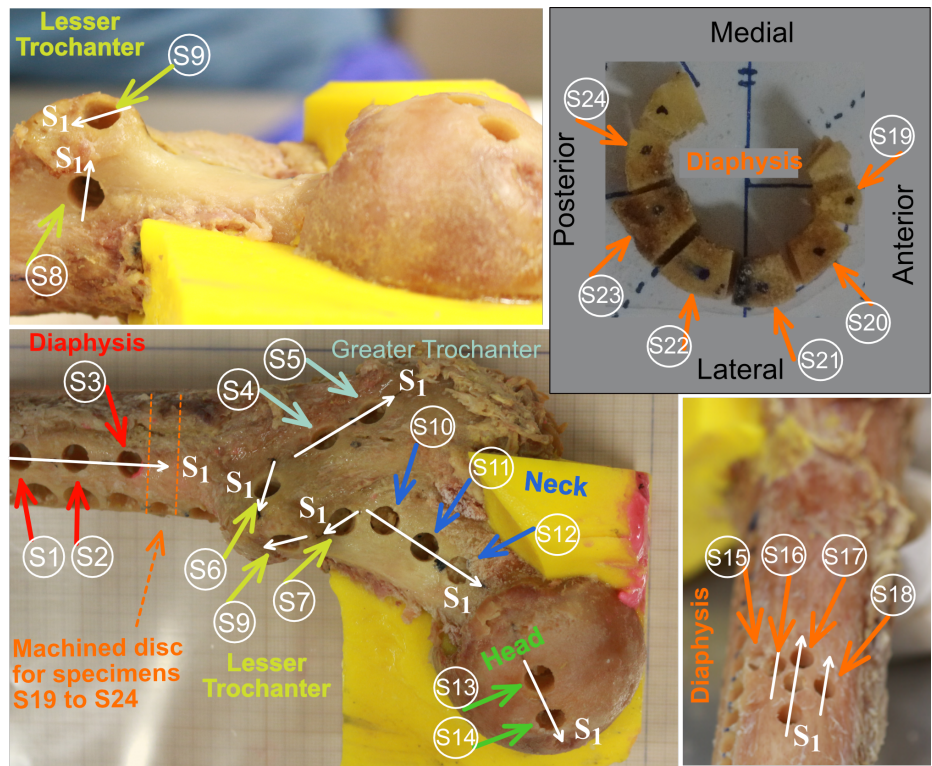

Figure 1. Extraction and position of the 24 specimens from the human left femur.

Diamond-tipped drills were used to machine specimens $S_{1}$ to $S_{18}$, which have a cylindrical shape with diameter $6 \mathrm{~mm}$ and height equal to the thickness of the cortical bone. Specimens $S_{19}$ to $S_{24}$ were manually cut and show a trapezoidal shape. During the cutting, water was used in order to reduce both friction and temperature rise. Before the extraction, an easily identifiable mark in the direction $S_{1}$ has been carved on the external surface of each specimen in order to orient it with respect to the femur (Figures 1 and 2). The direction $S_{1}$ is used to locate each specimen in the femur when the 3D microstructure is reconstructed from $\mu \mathrm{CT}$ images. Thus, it could be any direction. Here, for the sake of simplicity, we have

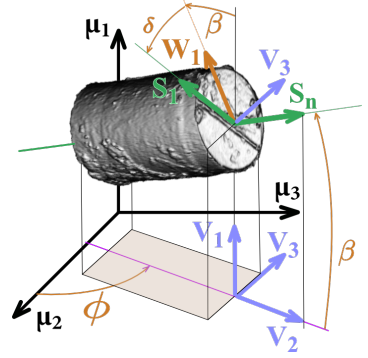

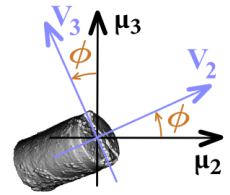

$\odot \mu_{1}$

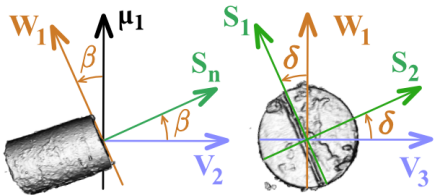

$\odot S_{n}$

Figure 2. Position of a specimen with respect to the $\mu \mathrm{CT}$ system of coordinates $R_{\mu}$. 

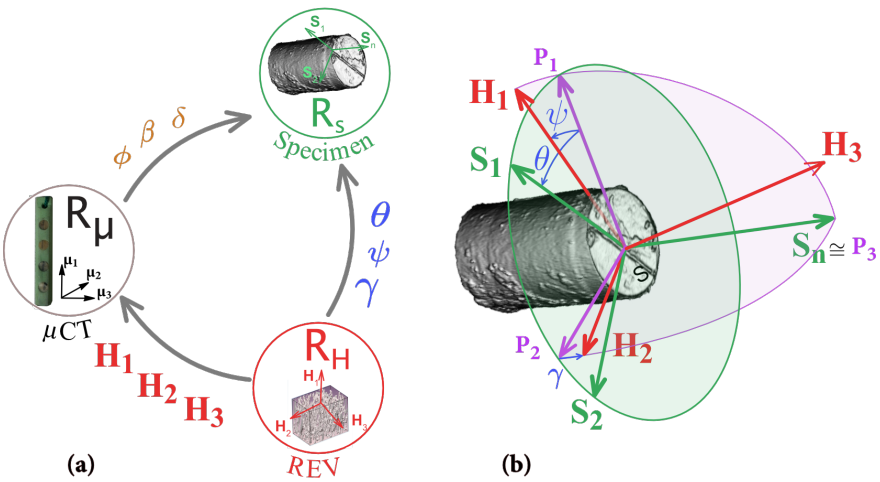

(b)

Figure 3. (a) Main steps to acquire the directions of orthotropy of the Haversian canals. (b) Angles defining the projection of $R_{H}$ on the external surface of the specimen.

decided to let it coincide with the middle line of the femur in each region of interest (Figure 1). Specimens were immersed in a solution of zinc iodide for 24 hours to stain the Haversian canals inside the osteons.

2.1.2. $\mu C T$ imaging. The specimens were placed on a shelf trying to align the direction $S_{1}$ with the vertical axis $\mu_{1}$ of the $\mu \mathrm{CT}$ scanner in the best possible way (Figure $3 b$ ). They were scanned using a $\mu \mathrm{CT}$ scanner (Scanco Medical XtremeCT with voxel size $7.4 \mu \mathrm{m}$ ). It consisted of a microfocus X-ray source, a rotating specimen holder, and a detector system, with a $2048 \times 2048$ pixel CCDD camera. The images were acquired using the following protocol: $90 \mathrm{kVp}, 155 \mu \mathrm{A}, 0.5 \mathrm{~mm}$ aluminum filter, and integration time $200 \mathrm{~ms}$ per slice.

After acquisition, a stack of about 800 cross-sectional images stored in DICOM format was obtained and the 3D reconstruction was made using software from FEI (Hillsboro, Oregon, USA). First, we built the 3D volume of the specimens in order to compute the outward normal vector $S_{n}$ to its external surface (Figure $3 b$ ). Second, by defining a specific threshold and by extracting one or more representative elementary volumes (REVs) for each specimen, we were able to obtain the 3D network of the Haversian canals. It is worth noting that an REV includes a sufficient number of osteons (i.e., at least 10, which corresponds to 3 to 4 osteons per side) and does not present any porosity which could trigger artifacts. In both cases (whole specimen and REV), the final 3D geometry was stored as an STL file constituted of a large number of facets $N_{f}\left(150000<N_{f}<200000\right)$ providing a uniform and smooth surface.

2.1.3. Identification of the main directions of the Haversian canals. In this section we detail the successive steps used to acquire the main directions of orthotropy 
associated with the Haversian canals (Figure 3a). Each system of reference used in this section is a direct orthonormal system of coordinates.

First, each specimen $S_{i}$ is defined by its proper system of reference $R_{S}=$ $\left\{S_{1}, S_{2}, S_{n}\right\}$, where the subscript $S$ stands for specimen, $S_{1}$ and $S_{n}$ were previously defined (Section 2.1.1), and $\boldsymbol{S}_{2}$ is obtained via a vector product between $\boldsymbol{S}_{1}$ and $S_{n}$ (Figure 3b). In order to determine the position of the specimen with respect to the $\mu \mathrm{CT}$ system of reference $R_{\mu}=\left\{\boldsymbol{\mu}_{1}, \boldsymbol{\mu}_{2}, \boldsymbol{\mu}_{3}\right\}$ (the subscript $\mu$ stands for $\mu \mathrm{CT}$ ), three angles are measured between $R_{s}$ and $R_{\mu}: \phi, \beta$, and $\delta$ (Appendix A1).

Second, once an REV is extracted from a specimen and using the geometrical information included in the STL files previously obtained (Section 2.1.2), the system of reference $R_{H}=\left\{\boldsymbol{H}_{1}, \boldsymbol{H}_{2}, \boldsymbol{H}_{3}\right\}$ (the subscript $H$ stands for Haversian canals) can be computed. To do so, rather than performing a topological analysis [Boyle and Kim 2011] of the REV surface mesh which would require an approximation of each Haversian canal by a regular geometry, we propose an approach which only takes into account the external surface of each Haversian canal while maintaining the precision, as demonstrated via simple illustrative examples in Section 3.1.

Each facet of the REV surface mesh is identified by its proper outward normal vector $\boldsymbol{n}_{j}$. Since for each REV the mesh facets have mostly the same area and their total number $N_{f}$ is high, no weighting has been applied. The product $\boldsymbol{n}_{j} \boldsymbol{n}_{j}^{T}$ enables one to obtain a tensorial form of $\boldsymbol{n}_{j}$, which includes more information than the vector itself (i.e., eigenvalues and eigenvectors). Then, by summing all these tensors, the global tensor $\boldsymbol{G}$ can be computed as

$$
\boldsymbol{G}=\sum_{j=1}^{N_{f}} \boldsymbol{n}_{j} \boldsymbol{n}_{j}^{T} .
$$

To quantify the morphology and the geometrical effects, we use the normalized eigenvalues $0 \leq \lambda_{k} \leq 3$, with $k \in[1,2,3]$, of $\boldsymbol{G}$, which are obtained from the eigenvalues $\lambda_{10} \leq \lambda_{20} \leq \lambda_{30}$ as

$$
\lambda_{k}=\frac{3 \lambda_{k 0}}{\lambda_{10}+\lambda_{20}+\lambda_{30}} .
$$

For each normalized eigenvalue $\lambda_{k}$, the associated eigenvector $\boldsymbol{H}_{k}$ can be calculated.

Finally, the projection of $R_{H}$ on the external surface of the specimen is computed to obtain the system of reference $R_{P}=\left\{\boldsymbol{P}_{1}, \boldsymbol{P}_{2}, \boldsymbol{P}_{3}\right\}$, where the subscript $P$ stands for projection (Appendix A2). The vectors $\boldsymbol{P}_{1}, \boldsymbol{P}_{2}$, and $\boldsymbol{P}_{3}$ are the projections of $\boldsymbol{H}_{1}, \boldsymbol{H}_{2}$, and $\boldsymbol{H}_{3}$, respectively (Figure $3 \mathrm{~b}$ ). Then, the position of $R_{H}$ for each REV with respect to $R_{S}$ can be found through three angles: $\psi, \gamma$, and $\theta$ (Figures $3 \mathrm{~b}$ and 4). The vector $\boldsymbol{P}_{1}$ will be directly compared to the corresponding numerical vector, which is obtained as described in the following sections. 

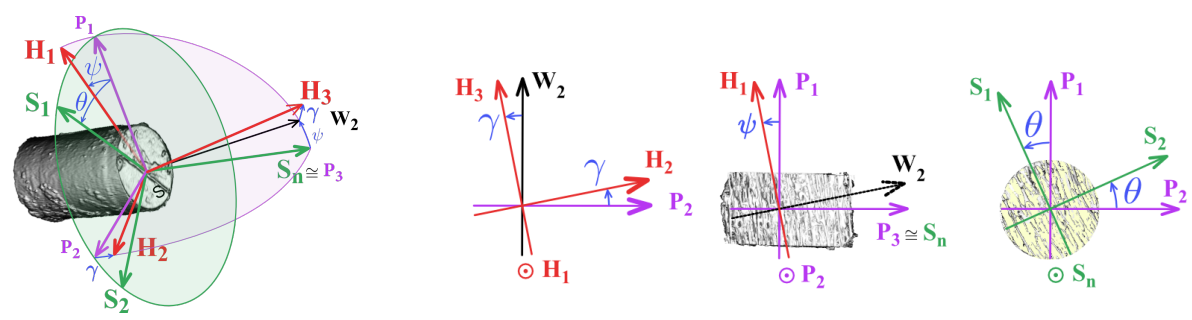

Figure 4. Angles defining the projection of $R_{H}$ on the external surface of the specimen ( $\boldsymbol{W}_{2}$ is an intermediate unit vector).

\section{Results}

3.1. Validation of the technique to identify the directions of orthotropy. To validate and illustrate our approach presented in Section 2.1.3, five simple examples, whose average direction $\boldsymbol{V}$ is known, are proposed as shown in Figure 5.

It has to be noticed that the cutting sections of the tubes are not proper surfaces of the tubes themselves but rather fictive ones obtained through the REV extraction. Therefore, for configurations (a) to (d), the upper and lower cutting planes are not taken into account. However, for the sake of practicality, for configuration (e) the extremities are included in the analysis.

For each configuration, the tubes are characterized by their direction $\boldsymbol{V}_{t 0}(t$ being the number of the tube in the specific configuration and going from 1 to $N_{t}$, the total number of tubes), which is defined in a spherical system of coordinates as $V_{t 0}=\left\{\cos \alpha_{t} \cos \gamma_{t}, \cos \alpha_{t} \sin \gamma_{t}, \sin \alpha_{t}\right\}$.

The average direction $\boldsymbol{V}$ of a configuration is then defined as

$$
\boldsymbol{V}=\frac{\sum_{l=1}^{N_{t}} \boldsymbol{V}_{t 0}}{\left\|\sum_{l=1}^{N_{t}} \boldsymbol{V}_{t 0}\right\|}
$$

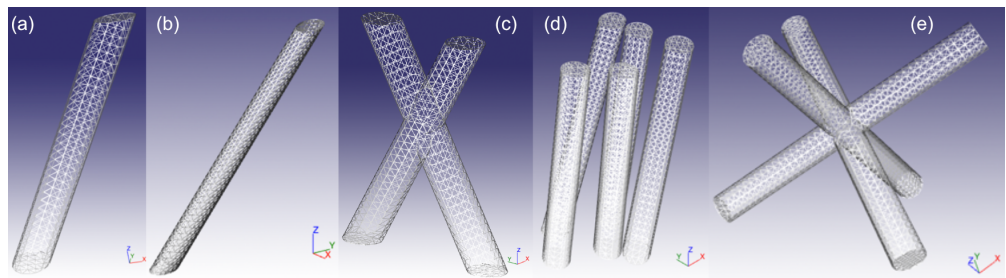

Figure 5. Simple examples to illustrate our approach to compute the geometric tensor $\boldsymbol{G}$. (a) Single tube with circular section. (b) Single tube with elliptical section. (c) Two crossed tubes. (d) Five noncrossed and randomly oriented tubes with circular section. (e) Three orthogonally crossed tubes. 


\begin{tabular}{|c|c|c|c|c|c|}
\hline & (a) & (b) & (c) & (d) & (e) \\
\hline$\alpha_{t} / \gamma_{t}$ & $60^{\circ} / 30^{\circ}$ & $60^{\circ} / 30^{\circ}$ & $\begin{array}{c}60^{\circ} / 90^{\circ} \\
60^{\circ} /-90^{\circ}\end{array}$ & $\begin{array}{l}50^{\circ} / 20^{\circ} \\
55^{\circ} / 25^{\circ} \\
60^{\circ} / 30^{\circ} \\
65^{\circ} / 35^{\circ} \\
70^{\circ} / 40^{\circ}\end{array}$ & $\begin{array}{c}0^{\circ} / 0^{\circ} \\
0^{\circ} / 90^{\circ} \\
90^{\circ} / 0^{\circ}\end{array}$ \\
\hline$V$ & $\begin{array}{l}0.433 \\
0.250 \\
0.866\end{array}$ & $\begin{array}{l}0.433 \\
0.250 \\
0.866\end{array}$ & $\begin{array}{l}0 \\
0 \\
1\end{array}$ & $\begin{array}{l}0.437 \\
0.237 \\
0.868\end{array}$ & \\
\hline $\begin{array}{l}\lambda_{1} \\
\lambda_{2} \\
\lambda_{3}\end{array}$ & $\begin{array}{l}0.010 \\
1.480 \\
1.510\end{array}$ & $\begin{array}{l}0.000 \\
0.990 \\
2.010\end{array}$ & $\begin{array}{l}0.320 \\
0.930 \\
1.750\end{array}$ & $\begin{array}{l}0.03 \\
1.420 \\
1.540\end{array}$ & $\begin{array}{l}0.998 \\
1.001 \\
1.001\end{array}$ \\
\hline $\boldsymbol{H}_{1}$ & $\begin{array}{l}0.432 \\
0.251 \\
0.866\end{array}$ & $\begin{array}{l}0.433 \\
0.250 \\
0.866\end{array}$ & $\begin{array}{r}-0.001 \\
0.001 \\
1.000\end{array}$ & $\begin{array}{l}0.408 \\
0.240 \\
0.881\end{array}$ & $\begin{array}{l}0.577 \\
0.577 \\
0.577\end{array}$ \\
\hline $\boldsymbol{H}_{2}$ & $\begin{array}{r}0.517 \\
0.718 \\
-0.466\end{array}$ & $\begin{array}{r}-0.501 \\
0.865 \\
0.001\end{array}$ & $\begin{array}{r}-0.013 \\
0.999 \\
-0.001\end{array}$ & $\begin{array}{r}0.598 \\
0.659 \\
-0.456\end{array}$ & $\begin{array}{r}-0.305 \\
-0.503 \\
0.808\end{array}$ \\
\hline Anisotropy mode & TI & $\mathrm{O}$ & $\mathrm{O}$ & TI & I \\
\hline
\end{tabular}

Table 1. Overall results for each configuration (a) to (e) $(\mathrm{TI}=$ transverse isotropy, $\mathrm{O}=$ orthotropy, and $\mathrm{I}=$ isotropy).

with $\|\cdot\|$ the Euclidean norm of a vector. The results associated with each configuration are reported in Table 1 . The direction $\boldsymbol{V}$ can be alternatively computed using the approach presented in Section 2.1.3. In fact, the first eigenvector $\boldsymbol{H}_{1}$ of the geometrical tensor $\boldsymbol{G}$ corresponds to $\boldsymbol{V}$. It is interesting to notice that an eigenvector $\boldsymbol{H}_{k}$ ( $k$ going from 1 to 3 ) is correctly computed only if the associated eigenvalue $\lambda_{k}$ is notably different from the other two. Thus, the direction $\boldsymbol{V}$ is properly estimated by the proposed approach for cases (a) to (d). However, for case (e), the eigenvalues $\lambda_{k}$ of $\boldsymbol{G}$ being identical and close to 1, the overall geometry is isotropic and the eigenvectors do not provide any further information. Similarly, the eigenvector $\boldsymbol{H}_{2}$ is indicative only if the eigenvalues $\lambda_{2}$ and $\lambda_{3}$ are different. This is not the case for configurations (a) and (d) where there is not a preferential transversal direction (i.e., transverse isotropy). Finally, for cases (b) and (c), the eigenvector $\boldsymbol{H}_{2}$ clearly shows the geometrical orthotropy.

The consistency of our technique has then been shown, and we can now apply it to the cortical bone. 


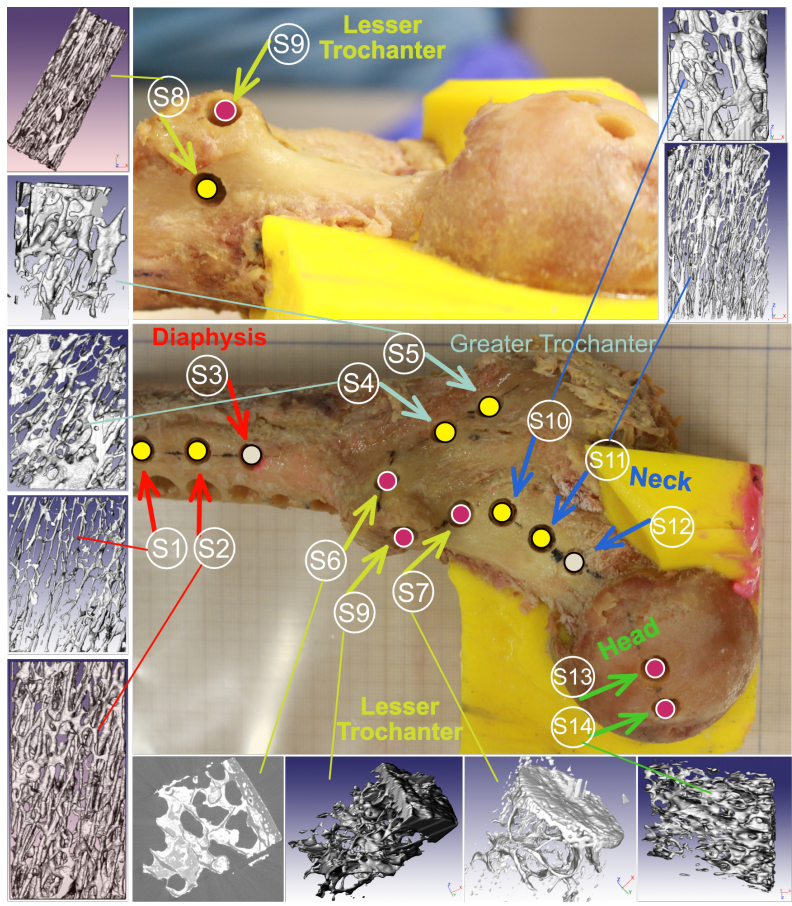

Figure 6. 3D reconstruction of the Haversian canals network for the REVs extracted from specimens $S_{1}, S_{2}, S_{4}, S_{5}, S_{8}, S_{10}$, and $S_{11}$ (yellow circles). For the other specimens (pink or gray circles), the reconstruction was not possible due to the very thin cortical thickness or some technical issues.

3.2. Measurement of the directions of orthotropy. For each of the 24 specimens the $3 \mathrm{D}$ geometry was reconstructed and its position with respect to the $\mu \mathrm{CT}$ system of coordinates $R_{\mu}$ was identified. In order to obtain the main direction $\boldsymbol{H}_{1}$ of the Haversian canals, at least one REV for each specimen was extracted where only Haversian canals (i.e., no large porosities) could be observed. Nevertheless, for some specimens (i.e., $S_{6}, S_{7}, S_{9}, S_{13}$, and $S_{14}$ ) only a few Haversian canals (i.e., fewer than five) could be segmented due to the very thin thickness of the cortical domain. Then, the orthotropic system of coordinates $R_{H}$ could not be computed, but we rather estimated the eigenvectors $\boldsymbol{H}_{k}$ of the geometric tensor $\boldsymbol{G}$ associated with the spongy trabeculae. Additionally, specimens $S_{3}, S_{6}$, and $S_{12}$ could not be retrieved due to some technical issues. In Figure 6, the REVs for specimens $S_{1}$, $S_{2}, S_{4}, S_{5}, S_{8}, S_{10}$, and $S_{11}$ are presented. In summary, we distinguish between the specimens with a cortical thickness $h_{C}$ greater (19, named cortical specimens) and less (5, named trabecular specimens) than $0.5 \mathrm{~mm}$. In the following sections, for each specimen we identify the eigenvalues $\left(\lambda_{1}, \lambda_{2}, \lambda_{3}\right)$ of the geometric tensor $\boldsymbol{G}$, 


\begin{tabular}{|c|c|c|c|c|c|c|c|c|c|c|c|}
\hline$S_{i}$ & REV & region & s.t. & $\lambda_{1}$ & $\lambda_{2}$ & $\lambda_{3}$ & $\begin{array}{c}\theta \\
\circ \pm 1\end{array}$ & $\begin{array}{c}\psi \\
\circ \\
\pm 1\end{array}$ & $\begin{array}{c}\gamma \\
\circ \pm 1\end{array}$ & $\begin{array}{c}h_{c} \\
\mathrm{~mm} \pm 0.4\end{array}$ & a.m. \\
\hline$S_{1}$ & $S_{1,1}$ & $\mathrm{AD}$ & $\mathrm{C}$ & 0.39 & 0.93 & 1.67 & -20.1 & 11.2 & -3.8 & & $\mathrm{O}$ \\
\hline$S_{2}$ & $S_{2,1}$ & $\mathrm{AD}$ & $\mathrm{C}$ & 0.46 & 1.12 & 1.42 & -9.9 & 2.0 & -35.5 & 4.1 & $\mathrm{O} / \mathrm{TI}$ \\
\hline$S_{3}$ & $S_{3,1}$ & $\mathrm{AD}$ & $\mathrm{C}$ & & & & & & & & LD \\
\hline$S_{4}$ & $S_{4,1}$ & GT & $\mathrm{C}$ & 0.42 & 1.06 & 1.52 & 31.3 & 1.1 & 0.9 & 0.8 & $\mathrm{O}$ \\
\hline$S_{5}$ & $S_{5,1}$ & GT & $\mathrm{C}$ & 0.38 & 0.76 & 1.86 & 20.5 & 3.0 & 10.4 & 0.8 & $\mathrm{O}$ \\
\hline$S_{6}$ & $S_{6,1}$ & LT & $\mathrm{T}$ & & & & & & & 0.5 & NVM \\
\hline$S_{7}$ & $S_{7,1}$ & LT & $\mathrm{T}$ & 0.43 & 0.72 & 1.80 & 33.0 & 2.9 & 5.7 & 0.4 & $\mathrm{O}$ \\
\hline$S_{8}$ & $S_{8,1}$ & LT & $\mathrm{C}$ & 0.35 & 1.01 & 1.64 & 87.5 & 0.2 & -3.5 & 2.5 & $\mathrm{O}$ \\
\hline$S_{9}$ & $S_{9,1}$ & LT & $\mathrm{T}$ & 0.62 & 0.99 & 1.39 & 70.8 & 9.8 & 83.9 & 0.5 & $\mathrm{O}$ \\
\hline$S_{10}$ & $S_{10,1}$ & $\mathrm{~N}$ & $\mathrm{C}$ & 0.37 & 0.74 & 1.90 & 20.5 & 7.3 & 3.9 & 0.7 & $\mathrm{O}$ \\
\hline$S_{11}$ & $S_{11,1}$ & $\mathrm{~N}$ & $\mathrm{C}$ & 0.37 & 1.14 & 1.48 & -2.3 & 5.2 & -0.2 & & $\mathrm{O}$ \\
\hline$S_{12}$ & $S_{12,1}$ & $\mathrm{~N}$ & $\mathrm{C}$ & & & & & & & & LD \\
\hline$S_{13}$ & $S_{13,1}$ & $\mathrm{H}$ & $\mathrm{T}$ & 0.43 & 1.04 & 1.53 & -96.1 & 80.5 & -69.1 & & $\mathrm{O}$ \\
\hline$S_{14}$ & $S_{14,1}$ & $\mathrm{H}$ & $\mathrm{T}$ & 0.43 & 1.08 & 1.49 & -89.2 & 79.7 & -60.9 & 0.5 & $\mathrm{O}$ \\
\hline$S_{15}$ & $S_{15,1}$ & PD & $\mathrm{C}$ & 0.76 & 1.11 & 1.14 & -7.5 & 7.9 & -53.2 & 5.3 & $\mathrm{TI}$ \\
\hline$S_{16}$ & $S_{16,1}$ & PD & $\mathrm{C}$ & 0.63 & 1.10 & 1.28 & -4.2 & 6.2 & 87.4 & 5.7 & $\mathrm{O} / \mathrm{TI}$ \\
\hline$S_{17}$ & $S_{17,1}$ & PD & $\mathrm{C}$ & 0.58 & 1.11 & 1.31 & 10.6 & 4.4 & -89.1 & 6.1 & $\mathrm{O} / \mathrm{TI}$ \\
\hline$S_{18}$ & $S_{18,1}$ & PD & $\mathrm{C}$ & 0.39 & 1.20 & 1.40 & 9.6 & 1.7 & -28.3 & 6.0 & $\mathrm{O} / \mathrm{TI}$ \\
\hline
\end{tabular}

Table 2. Overall results for specimens $S_{1}$ to $S_{18}$. Under s.t. (specimen type): $\mathrm{C}=$ cortical, $\mathrm{T}=$ trabecular. Under a.m. (anisotropy mode): $\mathrm{TI}=$ transverse isotropy, $\mathrm{O}=$ orthotropy, $\mathrm{I}=$ isotropy, $\mathrm{LD}=$ lost data, $\mathrm{NVM}=$ no visible mark.

the angles $\theta, \psi$, and $\gamma$, and the cortical thickness $h_{C}$. In Tables 2 and 3 all the results are reported. For the angles, the uncertainty of $1^{\circ}$ is due to the manual measurement. For the cortical thickness, an uncertainty of $0.4 \mathrm{~mm}$ corresponds to the largest transition region between the cortical and the spongy bone.

3.2.1. Cortical specimens. Although in reality there is no sharp transition between isotropy (I), transverse isotropy (TI), and orthotropy (O), here, in order to classify the degree of anisotropy for each specimen $S_{i}$, we have defined the intervals

- for $\mathrm{I}\left|\lambda_{i}-\lambda_{j}\right| \leq 0.05$,

- between I and TI $0.05<\left|\lambda_{i}-\lambda_{j}\right|<0.37$,

- for TI with respect to $\boldsymbol{H}_{1}\left|\lambda_{2}-\lambda_{1}\right| \geq 0.37$ and $\left|\lambda_{3}-\lambda_{2}\right| \leq 0.05$,

- between TI and $O\left|\lambda_{2}-\lambda_{1}\right| \geq 0.37$ and $0.05<\left|\lambda_{3}-\lambda_{2}\right|<0.37$, and

- for $\mathrm{O}\left|\lambda_{i}-\lambda_{j}\right| \geq 0.37$,

where $i, j \in\{1,2,3\}$ and $i \neq j$.

Overall, for each cortical specimen, $\lambda_{1}$ is much smaller than $\lambda_{2}$ and, more specifically, we found that specimens $S_{2}, S_{15}, S_{16}, S_{17}$, and $S_{18}$ show a transverse isotropy. 


\begin{tabular}{|c|c|c|c|c|c|c|c|c|c|c|c|}
\hline$S_{i}$ & REV & $\begin{array}{c}\chi \\
\circ{ }^{\circ} \\
\pm 1\end{array}$ & region & s.t. & $\lambda_{1}$ & $\lambda_{2}$ & $\lambda_{3}$ & $\begin{array}{c}\theta \\
\circ \pm 1\end{array}$ & $\begin{array}{c}\psi \\
{ }^{\circ} \pm 1\end{array}$ & $\begin{array}{l}\gamma \\
\circ \\
{ }^{\prime}\end{array}$ & a.m. \\
\hline \multirow{3}{*}{$S_{19}$} & $S_{19,1}$ & -11.3 & $\mathrm{D}$ & $\mathrm{C}$ & 0.62 & 1.06 & 1.30 & 8.4 & 1.8 & -2.5 & $\mathrm{O} / \mathrm{TI}$ \\
\hline & $S_{19,2}$ & -4.6 & $\mathrm{D}$ & $\mathrm{C}$ & 0.76 & 1.04 & 1.20 & 2.1 & -2.1 & -16.3 & $\mathrm{TI} / \mathrm{I}$ \\
\hline & $S_{19,3}$ & 5.4 & D & $\mathrm{C}$ & 0.68 & 0.97 & 1.35 & 3.6 & 1.6 & -4.7 & $\mathrm{O}$ \\
\hline \multirow{3}{*}{$S_{20}$} & $S_{20,1}$ & 33.4 & D & $\mathrm{C}$ & 0.64 & 1.03 & 1.33 & 16.3 & 7.3 & 12.0 & $\mathrm{O}$ \\
\hline & $S_{20,2}$ & 42.4 & $\mathrm{D}$ & $\mathrm{C}$ & 0.68 & 1.03 & 1.29 & 19.4 & 4.4 & -7.5 & $\mathrm{O} / \mathrm{TI}$ \\
\hline & $S_{20,3}$ & 51.8 & $\mathrm{D}$ & $\mathrm{C}$ & 0.59 & 1.10 & 1.31 & 16.8 & 1.8 & -26.3 & $\mathrm{O} / \mathrm{TI}$ \\
\hline \multirow{3}{*}{$S_{21}$} & $S_{21,1}$ & 70.0 & $\mathrm{D}$ & $\mathrm{C}$ & 0.62 & 1.08 & 1.30 & 11.7 & -0.6 & -36.1 & $\mathrm{O} / \mathrm{TI}$ \\
\hline & $S_{21,2}$ & 80.3 & $\mathrm{D}$ & $\mathrm{C}$ & 0.63 & 1.10 & 1.27 & 10.2 & -1.6 & -34.2 & $\mathrm{O} / \mathrm{TI}$ \\
\hline & $S_{21,3}$ & 87.7 & $\mathrm{D}$ & $\mathrm{C}$ & 0.53 & 1.21 & 1.26 & 8.6 & -1.9 & -71.3 & TI \\
\hline \multirow{5}{*}{$S_{22}$} & $S_{22,1}$ & 100.9 & $\mathrm{D}$ & $\mathrm{C}$ & 0.62 & 1.05 & 1.33 & 9.3 & 0.6 & -26.1 & $\mathrm{O} / \mathrm{TI}$ \\
\hline & $S_{22,2}$ & 118.2 & $\mathrm{D}$ & $\mathrm{C}$ & 0.61 & 1.13 & 1.26 & 9.5 & -0.4 & -5.6 & $\mathrm{O} / \mathrm{TI}$ \\
\hline & $S_{22,3}$ & 122.3 & $\mathrm{D}$ & $\mathrm{C}$ & 0.51 & 1.05 & 1.44 & 9.2 & 1.1 & -18.9 & $\mathrm{O}$ \\
\hline & $S_{22,4}$ & 126.9 & $\mathrm{D}$ & $\mathrm{C}$ & 0.68 & 1.05 & 1.27 & 18.0 & -1.1 & -17.9 & $\mathrm{O} / \mathrm{TI}$ \\
\hline & $S_{22,5}$ & 130.0 & $\mathrm{D}$ & $\mathrm{C}$ & 0.74 & 1.05 & 1.21 & 16.6 & -1.1 & -5.1 & $\mathrm{TI} / \mathrm{I}$ \\
\hline \multirow{3}{*}{$S_{23}$} & $S_{23,1}$ & 147.2 & $\mathrm{D}$ & $\mathrm{C}$ & 0.65 & 1.07 & 1.28 & 4.7 & -0.7 & -13.1 & $\mathrm{O} / \mathrm{TI}$ \\
\hline & $S_{23,2}$ & 160.2 & $\mathrm{D}$ & $\mathrm{C}$ & 0.68 & 0.99 & 1.32 & 1.3 & 0.6 & -4.8 & $\mathrm{O} / \mathrm{TI}$ \\
\hline & $S_{23,3}$ & 171.1 & D & $\mathrm{C}$ & 0.71 & 1.01 & 1.28 & -7.9 & 2.7 & -7.9 & $\mathrm{O} / \mathrm{TI}$ \\
\hline \multirow{3}{*}{$S_{24}$} & $S_{24,1}$ & 181.0 & D & $\mathrm{C}$ & 0.79 & 1.09 & 1.12 & -23.1 & -1.7 & -17.2 & TI \\
\hline & $S_{24,2}$ & 187.1 & $\mathrm{D}$ & $\mathrm{C}$ & 0.67 & 0.99 & 1.34 & -35.9 & -5.7 & -1.4 & $\mathrm{O}$ \\
\hline & $S_{24,3}$ & 197.6 & D & $\mathrm{C}$ & 0.68 & 1.06 & 1.26 & -28.6 & -16.1 & -22.9 & $\mathrm{O} / \mathrm{TI}$ \\
\hline
\end{tabular}

Table 3. Overall results for specimens $S_{19}$ to $S_{24}$. Under s.t. (specimen type): $\mathrm{C}=$ cortical. Under a.m. (anisotropy mode): $\mathrm{TI}=$ transverse isotropy, $\mathrm{O}=$ orthotropy, $\mathrm{I}=$ isotropy.

Then, in these cases, the angle $\gamma$, which generally provides the circumferential direction, is not relevant since $\lambda_{2} \approx \lambda_{3}$. The remaining cortical specimens (i.e., $S_{1}, S_{4}, S_{5}, S_{8}, S_{10}$, and $S_{11}$ ) are orthotropic. Around the diaphysis (i.e., from $S_{19}$ to $S_{24}$ ), some REVs clearly show an orthotropic behavior, whereas others are between orthotropy and transverse isotropy (Table 3 ).

It is interesting to focus on the specimens in the AD ( $S_{1}$ and $S_{2}$ ), in the PD (from $S_{15}$ to $S_{18}$ ), and around the diaphysis (from $S_{19}$ to $S_{24}$ ) for which $h_{c}>0.5$ and the Haversian canals are uniformly oriented. We found that the angle $\theta$ between the drawn mark $\boldsymbol{S}_{1}$ on each specimen and $\boldsymbol{P}_{1}$, the projection of the principal direction of the Haversian canals $\boldsymbol{H}_{1}$ on the external surface of the femur, varies between $-35.86^{\circ}$ for $S_{24,2}$ and $19.44^{\circ}$ for $S_{20,2}$.

We analyzed the evolution of $\theta$ along the radial direction (Figure 7). To do so, we split the reconstructed $S_{1}$ specimen into seven successive slices with a spacing of $0.5 \mathrm{~mm}$ (Figure 7) and we found $\theta$ equal to (a) $-43^{\circ}$, (b) $-22^{\circ}$, (c) $-15^{\circ}$, (d) 


\begin{tabular}{|c|cccc|}
\hline Image & $\lambda_{1}$ & $\lambda_{2}$ & $\lambda_{3}$ & $\theta\left(^{\circ}\right)$ \\
\hline Figure 7a & 0.39 & 0.55 & 1.09 & -42.7 \\
Figure 7b & 0.44 & 0.99 & 1.56 & -21.9 \\
Figure 7c & 0.40 & 0.98 & 1.61 & -15.0 \\
Figure 7d & 0.37 & 1.15 & 1.48 & -16.1 \\
Figure 7e & 0.39 & 1.22 & 1.39 & -16.6 \\
Figure 7f & 0.44 & 1.15 & 1.40 & -17.6 \\
Figure 7g & 0.44 & 1.18 & 1.38 & -14.9 \\
\hline
\end{tabular}

Table 4. Results for the extracted slices from specimen $S_{1}$.

$-16^{\circ}$, (e) $-17^{\circ}$, (f) $-18^{\circ}$, and $(\mathrm{g})-15^{\circ}$. If we consider that in the outermost slices (parts (a) and (b) of Figure 7) the presence of the external surface distorts the final outcome, one may conclude that $\theta$ does not change significantly when going from the outer to the inner cortical domain. The computed angles are reported in Table 4.

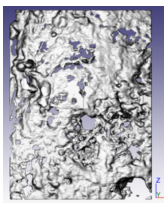

(a)

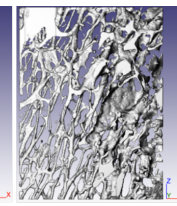

(b)

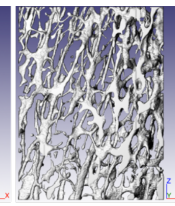

(c)

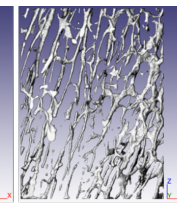

(d)

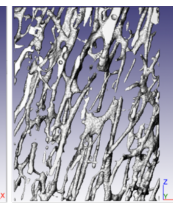

(e)

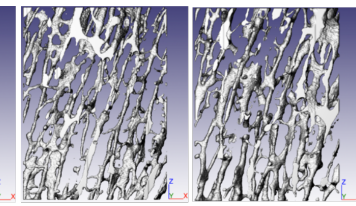

(f)

(g)

Figure 7. Successive slices from the exterior (a) to the interior (g) of an REV of specimen $S_{1}$.

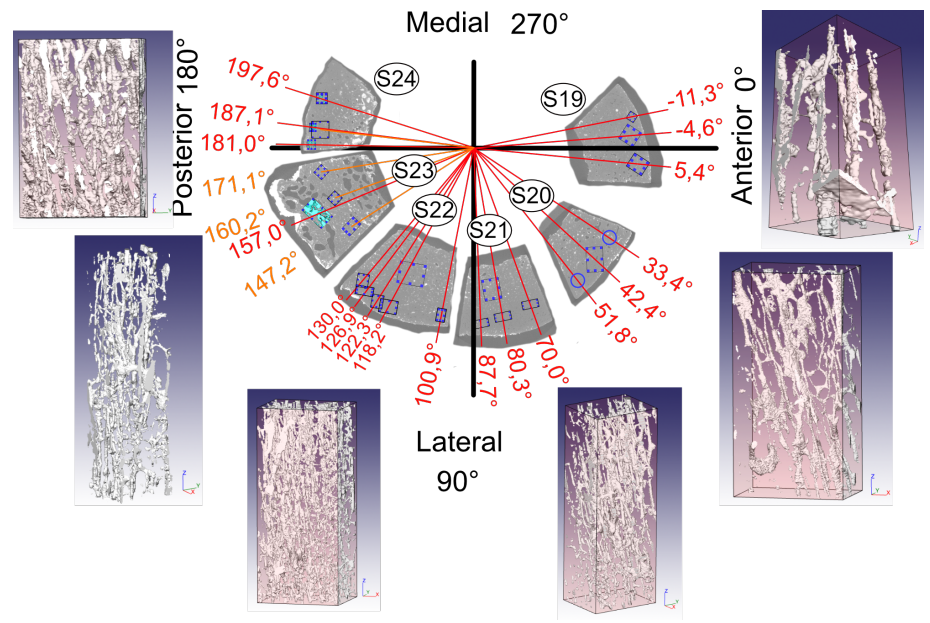

Figure 8. Specimens $S_{19}$ to $S_{24}$ with the relative circumferential position $\chi$ and some of the extracted REVs. 

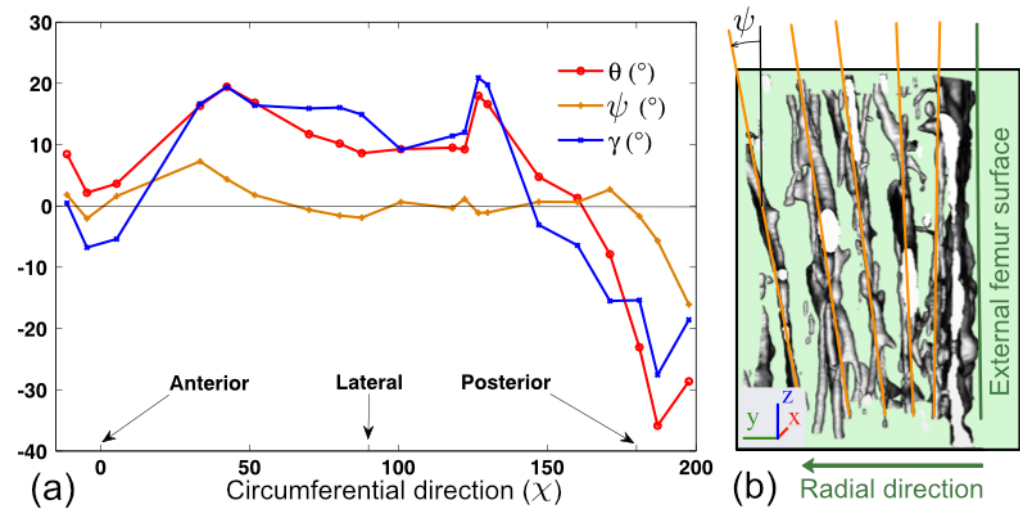

Figure 9. (a) Evolution of $\theta, \psi$, and $\gamma$ with respect to the circumferential position $\chi$ of the REVs in specimens $S_{19}$ to $S_{24}$. (b) Orientation of $\psi$ close to the external surface of the femur.

For specimens $S_{19}$ to $S_{24}$, twenty REVs were extracted, whose position around the diaphysis circumference is defined by the angle $\chi$, varying between $-11.3^{\circ}$ and $197.6^{\circ}$ (Figure 8 and Table 3). In Figure 9a, the evolutions of the angles $\theta$ (red line), $\psi$ (brown line), and $\gamma$ (blue line) around the upper diaphysis (i.e., from the anterior to the posterior region passing by the lateral region) are shown. We can notice that $\theta$ and $\gamma$ vary as functions of the circumferential coordinate $\chi$, whereas $\psi$ does not change significantly.

Finally, we investigated the variation of $\psi$, the angle between $\boldsymbol{P}_{1}$ and $\boldsymbol{H}_{1}$, across the cortical thickness along the radial direction for the specimen $S_{1}$. We found that, closer to the outer surface, the Haversian canals are nearly parallel to the surface (Figure 9b).

3.2.2. Trabecular specimens. As mentioned earlier, for some specimens $\left(S_{6}, S_{7}\right.$, $S_{9}, S_{13}$, and $S_{14}$ ) the cortical thickness is very thin. Thus, it is difficult to extract an REV with isolated Haversian canals and identify the average direction $\boldsymbol{H}_{1}$.

Although in the present work we focus on the orthotropy of the cortical bone, it is interesting to quantify the geometric tensor $\boldsymbol{G}$ and the associated variables for such specimens (Table 2). To do so, the parameters used for the reconstruction of the $3 \mathrm{D}$ geometries from the $\mu \mathrm{CT}$ images have been adapted in order to detect the trabeculae and larger REVs have been extracted.

Some remarks can be drawn. For specimens $S_{13}$ and $S_{14}$ extracted in the head, the angle $\psi$ is equal to $80.5^{\circ}$ and $79.7^{\circ}$, respectively. This means that the spongy trabeculae are mainly oriented perpendicular to the outer surface of the femur. On the contrary, for specimens $S_{7}$ and $S_{9}$ extracted in the neck, $\psi$ is found equal to $2.9^{\circ}$ and $9.8^{\circ}$, respectively, which implies that the trabeculae are almost parallel to 
the external femoral surface (Table 2). Such outcomes are in agreement with the literature and more specifically with the works of Jacobs et al. [1995] and Tsubota et al. [2009].

\section{Discussion}

Our approach is based on experimental data and more specifically on $\mu \mathrm{CT}$ images of the Haversian network, which provide the same trends found in [Petrtýl et al. 1996; Wirtz et al. 2003; Báča et al. 2007]. We have defined a geometric tensor $\boldsymbol{G}$, via the outward normal vectors to the facets of an REV, and we have computed its eigenvalues and eigenvectors to estimate the degree and the directions of orthotropy, respectively.

Despite the interesting results, such a technique shows few main drawbacks. First, the position of each specimen with respect to the $\mu \mathrm{CT}$ system of coordinates $R_{\mu}$ needs to be carefully retrieved in order to obtain consistent results. Second, it is a discrete approach since the measurements are obtained via a series of specimens in specific regions of the 3D structure. In order to circumvent this issue, we propose in a further work a numerical technique based on diffusion equations and on the experimental data obtained in this paper. This approach will allow us to get a continuum description of the field of orthotropic directions in a 3D structure such a femur.

\section{Appendix}

A1. Position of $\boldsymbol{R}_{S}$ with respect to $\boldsymbol{R}_{\boldsymbol{\mu}}$. As explained in Section 2.1.3, it is necessary to determine the position of a specimen $S_{i}$ with respect to the $\mu \mathrm{CT}$. Each specimen is identified by the mark $S_{1}$ and the outward normal vector $S_{n}$, which constitute with $S_{2}$ the system of coordinates $R_{S}=\left\{S_{1}, S_{2}, S_{n}\right\}$. During the image acquisition, the position of a specimen with respect to the $\mu \mathrm{CT}$ system of coordinates $R_{\mu}=\left\{\boldsymbol{\mu}_{1}, \boldsymbol{\mu}_{2}, \boldsymbol{\mu}_{3}\right\}$ is defined by three angles (Figure 2 ):

- $\phi$, the angle between $\boldsymbol{\mu}_{2}$ and $\boldsymbol{V}_{2}$,

- $\beta$, the angle between $\boldsymbol{V}_{2}$ and $\boldsymbol{S}_{n}$, and

- $\delta$, the angle between $\boldsymbol{W}_{1}$ and $\boldsymbol{S}_{1}$,

where $R_{v}=\left\{V_{1}, V_{2}, V_{3}\right\}$ is an intermediate system of reference obtained by projecting the specimen on the plane $\left(\boldsymbol{\mu}_{2}, \boldsymbol{\mu}_{3}\right)$. Then, $\boldsymbol{V}_{1}=\boldsymbol{\mu}_{1}, \boldsymbol{V}_{2}$ is the normalized projection of $S_{n}$ on the plane $\left(\boldsymbol{\mu}_{2}, \boldsymbol{\mu}_{3}\right)$, and $\boldsymbol{V}_{3}$ is the cross product between $\boldsymbol{V}_{1}$ and $\boldsymbol{V}_{2}$. Finally, the vector $\boldsymbol{W}_{1}$ is the normalized projection of $\boldsymbol{V}_{1}$ on the specimen external surface.

A2. Projection of $R_{H}$ on the external surface of a specimen. As described in Section 2.1.3, it is necessary to identify the position of the Haversian canals with 
respect to the specimen. When an REV is extracted from a specimen, the system of orthotropic coordinates $R_{H}=\left\{\boldsymbol{H}_{1}, \boldsymbol{H}_{2}, \boldsymbol{H}_{3}\right\}$ of the Haversian canals can be determined. The projection of $R_{H}$ on the external surface of the specimen is computed and the system of reference $R_{P}$ is obtained. Then, three angles define the position of $R_{H}$ with respect to $R_{S}$ (Figure 4):

- $\theta$, the angle between $\boldsymbol{P}_{1}$ and $\boldsymbol{S}_{1}$, expressed as

$$
\theta=-\arcsin \left(\boldsymbol{P}_{1}^{T} \boldsymbol{S}_{2}\right)
$$

- $\psi$, the angle between $\boldsymbol{H}_{1}$ and $\boldsymbol{P}_{1}$, which reads

$$
\psi=-\arcsin \left(\boldsymbol{S}_{n}^{T} \boldsymbol{H}_{1}\right)
$$

and

- $\gamma$, the angle between $\boldsymbol{H}_{2}$ and $\boldsymbol{P}_{2}$, defined as

$$
\gamma=-\arcsin \left(\boldsymbol{P}_{2}^{T} \boldsymbol{H}_{3}\right)
$$

where $\left(\boldsymbol{c}^{T} \boldsymbol{d}\right)$ indicates the dot product, the superscript $T$ the transpose of a vector, and

$$
\begin{aligned}
& \boldsymbol{P}_{2}=\frac{\boldsymbol{H}_{1} \wedge \boldsymbol{S}_{n}}{\left\|\boldsymbol{H}_{1} \wedge \boldsymbol{S} n\right\|}, \\
& \boldsymbol{P}_{1}=\frac{\boldsymbol{S}_{n} \wedge \boldsymbol{P}_{2}}{\left\|\boldsymbol{S}_{n} \wedge \boldsymbol{P}_{2}\right\|}
\end{aligned}
$$

with $(\boldsymbol{c} \wedge \boldsymbol{d})$ the vector product. It is worth noting that in (4), (5), and (6), rather than simply computing the arc cosine between the two involved vectors, the arc sine is used in order to detect the sign of the angle of interest.

\section{Acknowledgements}

The authors are grateful to the ParisTech BiomecAM chair program on subjectspecific musculoskeletal modeling for partial funding of the work and to Sonia Simoes for her support during $\mu \mathrm{CT}$ imaging.

\section{References}

[Báča et al. 2007] V. Báča, D. Kachlík, Z. Horák, and J. Stingl, "The course of osteons in the compact bone of the human proximal femur with clinical and biomechanical significance", Surg. Radiol. Anat. 29:3 (2007), 201-207.

[Bernard et al. 2013] S. Bernard, Q. Grimal, and P. Laugier, "Accurate measurement of cortical bone elasticity tensor with resonant ultrasound spectroscopy”, J. Mech. Behav. Biomed. 18 (2013), $12-19$. 
[Boyle and Kim 2011] C. Boyle and I. Y. Kim, "Three-dimensional micro-level computational study of Wolff's law via trabecular bone remodeling in the human proximal femur using design space topology optimization", J. Biomech. 44:5 (2011), 935-942.

[Giorgio et al. 2017] I. Giorgio, U. Andreaus, F. dell'Isola, and T. Lekszycki, "Viscous second gradient porous materials for bones reconstructed with bio-resorbable grafts", Extreme Mech. Lett. 13 (2017), 141-147.

[Heřt et al. 1994] J. Heřt, P. Fiala, and M. Petrtýl, "Osteon orientation of the diaphysis of the long bones in man”, Bone 15:3 (1994), 269-277.

[Jacobs et al. 1995] C. R. Jacobs, M. E. Levenston, G. S. Beaupré, J. C. Simo, and D. R. Carter, "Numerical instabilities in bone remodeling simulations: the advantages of a node-based finite element approach", J. Biomech. 28:4 (1995), 449-451.

[Nazemi et al. 2016] S. M. Nazemi, D. M. L. Cooper, and J. D. Johnston, "Quantifying trabecular bone material anisotropy and orientation using low resolution clinical CT images: a feasibility study", Med. Eng. Phys. 38:9 (2016), 978-987.

[Petrtýl et al. 1996] M. Petrtýl, J. Heřt, and P. Fiala, "Spatial organization of the haversian bone in man", J. Biomech. 29:2 (1996), 161-169.

[Placidi et al. 2017] L. Placidi, U. Andreaus, and I. Giorgio, "Identification of two-dimensional pantographic structure via a linear D4 orthotropic second gradient elastic model", J. Eng. Math. 103 (2017), 1-21.

[Pothuaud et al. 2000] L. Pothuaud, P. Porion, E. Lespessailles, C. L. Benhamou, and P. Levitz, "A new method for three-dimensional skeleton graph analysis of porous media: application to trabecular bone microarchitecture", J. Microsc. 199:2 (2000), 149-161.

[Rémond et al. 2016] Y. Rémond, S. Ahzi, M. Baniassadi, and H. Garmestani, Applied RVE reconstruction and homogenization of heterogeneous materials, Wiley, 2016.

[Rho 1996] J.-Y. Rho, "An ultrasonic method for measuring the elastic properties of human tibial cortical and cancellous bone", Ultrasonics 34:8 (1996), 777-783.

[Rho et al. 1998] J.-Y. Rho, L. Kuhn-Spearing, and P. Zioupos, "Mechanical properties and the hierarchical structure of bone", Med. Eng. Phys. 20:2 (1998), 92-102.

[Tsubota et al. 2009] K.-i. Tsubota, Y. Suzuki, T. Yamada, M. Hojo, A. Makinouchi, and T. Adachi, "Computer simulation of trabecular remodeling in human proximal femur using large-scale voxel FE models: approach to understanding Wolff's law”, J. Biomech. 42:8 (2009), 1088-1094.

[Wirtz et al. 2003] D. C. Wirtz, T. Pandorf, F. Portheine, K. Radermacher, N. Schiffers, A. Prescher, D. Weichert, and F. U. Niethard, "Concept and development of an orthotropic FE model of the proximal femur”, J. Biomech. 36:2 (2003), 289-293.

Received 9 Apr 2018. Revised 13 Jun 2018. Accepted 29 Jul 2018.

Christophe Cluzel: cluzel@lmt.ens-cachan.fr

Laboratoire de Mécanique et Technologie, Ecole Normale Supérieure Paris Saclay, Cachan, France

RACHELE ALLENA: rachele.allena@ensam.eu

Institute de Biomécanique Humaine George Charpak, Arts et Métiers ParisTech, Paris, France 
EDITORIAL BOARD

ANTONIO CARCATERRA

ERIC A. CARLEN

FRANCESCO DELL'ISOLA

RAFFAELE ESPOSITO

ALBERT FANNJIANG

Gilles A. FrancFort

PieRANGelo MARCATI

JEAN-JACQUES MARIGO

PETER A. MARKOWICH

MARTIN OSTOJA-STARZEWSKI

PIERRE SEPPECHER

DAVID J. STEIGMANN

PAUl STEINMANN

PierRe M. SuQueT

MANAGING EDITORS

MICOL AMAR

CORRADO LATTANZIO

ANGELA MADEO

MARTIN OSTOJA-STARZEWSKI

ADVISORY BOARD

ADNAN AKAY

Holm AltenbaCH

MICOL AMAR

HARM ASKES

TEODOR ATANACKOVIĆ

VICTOR BERDICHEVSKY

GUY BOUCHITTÉ

ANDREA BRAIDES

ROBERTO CAMASSA

MAURO CARFORE

ERIC DARVE

FELIX DARVE

ANNA DE MASI

GianPiETRo DEL PIERo

EMmANUELE Di BENEDETTO

BERNOLD FIEDLER

IRENE M. GAMBA

DAVID Y. GAO

SERGEY GAVRILYUK

Timothy J. HEALEY

DOMINIQUE JEULIN

ROGER E. KHAYAT

CORRADO LATTANZIO

ROBERT P. LIPTON

ANGELO LUONGO

ANGELA MADEO

JUAN J. MANFREDI

CARlo MARCHIORO

ROBERTO NATALINI

PATRIZIO NEFF

ANDREY PIATNITSKI

ERRICO PRESUTtI

MARIO PULVIRENTI

LuCiO RuSSO

Miguel A. F. SANJUAN

PATRICK SElVAdURAI

ALEXANDER P. SEYRANIAN

MiroslaV ŠILHAVÝ

GUIDO SWEERS

ANTOINETTE TORDESILLAS

LEV TRUSKINOVSKY

JUAN J. L. VELÁZQUEZ

VINCENZO VESPRI

ANGELO VULPIANI msp.org/memocs

Università di Roma "La Sapienza", Italia

Rutgers University, USA

(CO-CHAIR) Università di Roma "La Sapienza", Italia

(TREASURER) Università dell'Aquila, Italia

University of California at Davis, USA

(CO-CHAIR) Université Paris-Nord, France

Università dell' Aquila, Italy

École Polytechnique, France

DAMTP Cambridge, UK, and University of Vienna, Austria

(CHAIR MANAGING EDITOR) Univ. of Illinois at Urbana-Champaign, USA

Université du Sud Toulon-Var, France

University of California at Berkeley, USA

Universität Erlangen-Nürnberg, Germany

LMA CNRS Marseille, France

Università di Roma "La Sapienza", Italia

Università dell' Aquila, Italy

Université de Lyon-INSA (Institut National des Sciences Appliquées), France

(CHAIR MANAGING EDITOR) Univ. of Illinois at Urbana-Champaign, USA

Carnegie Mellon University, USA, and Bilkent University, Turkey

Otto-von-Guericke-Universität Magdeburg, Germany

Università di Roma "La Sapienza", Italia

University of Sheffield, UK

University of Novi Sad, Serbia

Wayne State University, USA

Université du Sud Toulon-Var, France

Università di Roma Tor Vergata, Italia

University of North Carolina at Chapel Hill, USA

Università di Pavia, Italia

Stanford University, USA

Institut Polytechnique de Grenoble, France

Università dell'Aquila, Italia

Università di Ferrara and International Research Center MEMOCS, Italia

Vanderbilt University, USA

Freie Universität Berlin, Germany

University of Texas at Austin, USA

Federation University and Australian National University, Australia

Université Aix-Marseille, France

Cornell University, USA

École des Mines, France

University of Western Ontario, Canada

Università dell' Aquila, Italy

Louisiana State University, USA

Università dell'Aquila, Italia

Université de Lyon-INSA (Institut National des Sciences Appliquées), France University of Pittsburgh, USA

Università di Roma "La Sapienza", Italia

Istituto per le Applicazioni del Calcolo "M. Picone", Italy

Universität Duisburg-Essen, Germany

Narvik University College, Norway, Russia

Università di Roma Tor Vergata, Italy

Università di Roma "La Sapienza", Italia

Università di Roma "Tor Vergata”, Italia

Universidad Rey Juan Carlos, Madrid, Spain

McGill University, Canada

Moscow State Lomonosov University, Russia

Academy of Sciences of the Czech Republic

Universität zu Köln, Germany

University of Melbourne, Australia

École Polytechnique, France

Bonn University, Germany

Università di Firenze, Italia

Università di Roma La Sapienza, Italia

MEMOCS (ISSN 2325-3444 electronic, 2326-7186 printed) is a journal of the International Research Center for the Mathematics and Mechanics of Complex Systems at the Università dell'Aquila, Italy.

Cover image: "Tangle" by @ John Horigan; produced using the Context Free program (contextfreeart.org).

\section{PUBLISHED BY}

mathematical sciences publishers nonprofit scientific publishing

http://msp.org/

(C) 2018 Mathematical Sciences Publishers 
Mathematics and Mechanics of Complex Systems vol. 6 no. 4

A model for interfaces and its mesoscopic limit

Michele Aleandri and Venanzio Di Giulio

Optimal orthotropy and density distribution of two-dimensional structures

Narindra Ranaivomiarana, François-Xavier Irisarri, Dimitri

Bettebghor and Boris Desmorat

A multiphysics stimulus for continuum mechanics bone remodeling 307

Daniel George, Rachele Allena and Yves Rémond

On linear non-local thermo-viscoelastic waves in fluids

Joe D. Goddard

Heterogeneous directions of orthotropy in three-dimensional

structures: finite element description based on diffusion equations

Rachele Allena and Christophe Cluzel

A general method for the determination of the local orthotropic directions of heterogeneous materials: application to bone structures using $\mu \mathrm{CT}$ images

Christophe Cluzel and Rachele Allena

MEMOCS is a journal of the International Research Center for the Mathematics and Mechanics of Complex Systems at the Università dell' Aquila, Italy.

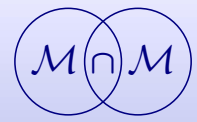

